\title{
Predictive value of left atrial appendage lobes on left atrial thrombus or spontaneous echo contrast in patients with non-valvular atrial fibrillation
}

Fan Wang ${ }^{\dagger}$, Mengyun Zhu ${ }^{\dagger}$, Xiaoyu Wang ${ }^{\dagger}$, Wei Zhang ${ }^{\dagger}$, Yang Su, Yuyan Lu, Xin Pan, Di Gao, Xianling Zhang, Wei Chen, Yawei Xu, Yuxi Sun ${ }^{*}$ and Dachun $X^{*}{ }^{*}$ (i)

\begin{abstract}
Background: Left atrial appendage morphology has been proved to be an important predictor of left atrial thrombus (LAT) and left atrial spontaneous echo contrast (LASEC) and stroke in patients with non-valvular atrial fibrillation (NVAF). However, the relation between left atrial appendage (LAA) lobes and LAT or LASEC is still unknown. The aim of this study is to investigate the correlation between the number of left atrial appendage lobes and LAT/LASEC in patients with NVAF.

Methods: This monocentric cross-sectional study enrolled 472 consecutive patients with non-valvular atrial fibrillation, who had transthoracic echocardiography (TTE) and transesophageal echocardiography (TEE) prior to cardioversion or left atrial appendage closure (LAAC) from July 2009 to August 2015 in department of cardiology of Shanghai Tenth People's Hospital. Patients who had significant mitral or aortic valve disease, previous cardiac valvular surgery and other complicated cardiac diseases were excluded. Individuals were divided into two groups:the LAT/LASEC group (16.95\%), which comprised patients with LAT or LASEC, as confirmed by TEE; and a negative control group (83.05\%).Baseline overall group characterization with demographic, clinical, laboratory data and echocardiographic parameters, alongside with information on medication was obtained for all patients. Subgroup analysis with line chart was applied for exploring the association between LAA lobes and LAT/LAESC. Receptor-operating curves (ROC) were used to test the value of LA anteroposterior diameter detected by different echocardiography methods predicting LAT or LASEC. Multivariable logistic regression analysis was used to investigate independent predictors of LAT/LASEC.

(Continued on next page)
\end{abstract}

\footnotetext{
* Correspondence: zhggsmlsyx@163.com; 1158009156@qq.com

${ }^{\dagger}$ Fan Wang, Mengyun Zhu, Xiaoyu Wang and Wei Zhang contributed equally

to this work.

Department of Cardiology, Shanghai Tenth People's Hospital, Tongji

University School of Medicine, NO. 301 Middle Yanchang Road, Shanghai

200072, China
}

(c) The Author(s). 2018 Open Access This article is distributed under the terms of the Creative Commons Attribution 4.0 International License (http://creativecommons.org/licenses/by/4.0/), which permits unrestricted use, distribution, and reproduction in any medium, provided you give appropriate credit to the original author(s) and the source, provide a link to the Creative Commons license, and indicate if changes were made. The Creative Commons Public Domain Dedication waiver (http://creativecommons.org/publicdomain/zero/1.0/) applies to the data made available in this article, unless otherwise stated. 


\begin{abstract}
(Continued from previous page)
Results: Among 472 patients, 23 (4.87\%) had LA/LAA thrombus and 57 (12.1\%) had LA spontaneous echo contrast. Compared to the negative group, patients in LAT/LASEC group had higher $\mathrm{CHA}_{2} \mathrm{DS}_{2}$-VASc score $(3.79 \pm 1.75 \mathrm{vs} 2.65 \pm$ $1.76, p<0.001$ ), larger LAD (measured by TTE, $48.1 \pm 7.7$ vs $44.6 \pm 6.5, P<0.001$; measured by TEE, $52.2 \pm 6.2$ vs $46.7 \pm 7.1$, $P<0.001$ ), lower left upper pulmonary venous flow velocity (LUPVFV $(0.54 \pm 0.17 \mathrm{~m} / \mathrm{s}$ vs $0.67 \pm 0.26 \mathrm{~m} / \mathrm{s}, \mathrm{Cl} 95 \% 0.05-0.22$, $P=0.003)$, more left atrial appendage lobes $(1.67 \pm 0.77$ vs $1.25 \pm 0.50, p<0.001)$. There was a good discriminative capacity for LAD detected by TTE (area under the curve (AUC), 0.67, Cl 95\% 0.61-0.73, $p<0.001$ ) and LAD detected by TEE (AUC, $0.73, \mathrm{Cl} 95 \% 0.67-0.79, p<0.001)$. The subgroup analysis based on gender and different LAA lobes yielded similar results (male group: $p<0.001$;female group: $p=0.004$ ) that the number of LAA lobes were significantly associated with LA thrombus or SEC. In multivariable logistic regression analysis, both the number of LAA lobes (odds ratio: 2.37; CI 95\% 1.37-4.09; $p=0.002$ ) and the persistent $\mathrm{AF}$ (odds ratio: 3.57; Cl 95\% 1.68-7.57; $p=0.001$ ) provided independent and incremental predictive value beyond $\mathrm{CHA}_{2} \mathrm{DS}_{2}$-VASc score.
\end{abstract}

Conclusion: The number of LAA lobes is an independent risk factor and has a moderate predictive value for LAT/LASEC among NVAF patients in China.

Keywords: Left atrial appendage, Left atrial Thrombus, Left atrial spontaneous echo contrast, Atrial fibrillation

\section{Background}

Atrial fibrillation (AF) is one of the most commonly observed arrhythmias in clinical practice with an incidence of $0.77 \%$ in China and approximately $1.5-2.0 \%$ in the developed world. This arrhythmia is associated with a five-fold risk of stroke, and 20-30\% of all strokes are due to AF, thus a higher mortality compared with those without AF [1-3]. Actually, over $90 \%$ of embolic stroke was caused by thrombi that originating from left atrial appendage (LAA) [4, 5]. LAA was described as a long, narrow, tubular, wavy, hooked structure with different lobes and a narrow junction and crenellated lumen [6, 7], creating a favorable condition for thrombosis, especially under the situation of AF. Thus it is of great importance to identify the thrombi or signs indicating thrombi formation in LAA. Presence of thrombus, spontaneous echo contrast (SEC) in LA/LAA, or decreased LAA emptying velocity has been reported as markers of thromboembolic risk in non-valvular atrial fibrillation (NVAF) [8-10]. Thrombus was defined as a hyperechogenic non-muscular and non-endocardial mass detected by more than one plane axis during transesophageal echocardiography (TEE), and SEC was defined as smoke-like material with a characteristic swirling motion that persisted throughout the cardiac cycle [11, 12].Actually, the severity of LASEC quantified by different semi-quantitative assessments has been proven to be associated with stroke events in patients with NVAF. It's reported that denser LASEC was accompanied by a higher risk of LAA thrombus formation in patients with NVAF [13-15]. However, in our study, we don't pay attention to semi-quantitative methods of LASEC which are largely influenced by the experience of the operator. We look LAA thrombus as the densest LASEC. The presence of at least one of them was designated left atrial abnormality.
Remarkably, it is observed that even in patients with AF, the incidence of AF associated stroke varied widely, ranging from 1 to $20 \%$ annually [4]. One possible mechanism behind may be that the incidence of thrombi formation in LAA with different anatomical characteristics, i.e., LAA morphology and number of LAA lobes, varied. Now it is recognized that there were four LAA macroscopic morphologies, including cactus LAA, chicken wing LAA, windsock LAA and cauliflower LAA $[5,16-$ 21]. Several recent studies have demonstrated that different LAA morphologies obtained by Cardiac CT or MRI are closely correlated with LASEC, transient ischaemic attacks (TIA) and strokes in patients with AF [16-21]. However, the relationship between LAA lobes and markers of thromboembolic risk (decreased LAA flow velocity, LASEC, LA thrombus) has not been fully characterized in patients with NVAF. Therefore, the aim of this study is to examine whether the number of left atrial appendage lobes could influence the development of left atrial thrombus (LAT) or left atrial spontaneous echo contrast (LASEC) in patients with NVAF.

\section{Methods}

\section{Study design}

We studied 472 patients with all types of non-vavular AF. Left atrial thrombus and spontaneous echo contrast were analyzed by $2 \mathrm{D}$-TEE and classified into two groups (LAT or LASEC positive group and negative group). Simultaneously, LAA lobes were counted during TEE procedure. Then, univariate analysis was performed using the Student's t-test and chi-square test. Predictors from univariate analysis were used for obtaining logistic regression models that could determine the relative importance of independent predictors of LAAT and LASEC [22]. 


\section{Enrollment}

This single-center cross-sectional study enrolled patients undergoing TTE and TEE prior to catheter ablation or left atrial appendage closure (LAAC) during a non-valvular AF episode. A total of 472 consecutive participants (males, 57.4\%; mean age, $66.1 \pm 10.8$ years) who were hospitalized at Department of Cardiology of Shanghai Tenth People's Hospital of Tongji University from July 2009 to August 2015 were referred to our center. AF was identified by an electrocardiogram and met the diagnostic criteria used in 2011 ACCF/AHA/HRS Guidelines for the management of patients with AF [1]. Exclusion criteria included: [i] moderate or severe mitral stenosis; [ii] severe mitral regurgitation; [iii] severe aortic stenosis; [iv] prosthetic mitral or aortic valves; [v] patients with unsuitable images for accurate assessment of TEE surrogate markers of stroke; [vi] congenital heart disease (i.e. atrial septal defect, ventricular septal defect, et al...); [vii] any contraindication to TEE (i.e. esophageal obstruction, esophageal varices, et al); [vii] poor image quality. Then all of these patients were stratified into two subgroups based on with or without LAT or LASEC.

\section{Initial data collection}

All individuals were subjected to thorough history taking and full clinical evaluation. Patient gender, age, heart rate, systolic blood pressure (SBP), diastolic blood pressure (DBP), type of AF, duration of $\mathrm{AF}, \mathrm{CHA}_{2} \mathrm{DS}_{2}$-VASc score, antiplateletdrugs or anticoagulant drugs, antiarrhythmic drugs, as well as history of congestive heart failure, hypertension, diabetes mellitus, previous stroke, vascular disease and other related diseases, were recorded. $\mathrm{CHA}_{2} \mathrm{DS}_{2}$-VASc score were calculated with 1 point assigned for a history of congestive heart failure, hypertension, 74 years $\geq$ age $\geq 65$ years, female, diabetes mellitus and vascular disease and 2 points assigned for age $\geq 75$ years, a history of stroke or transient ischemic attack (TIA), the maximum score is 9. Specifically, previous stroke also included lacunar infarction.

\section{Echocardiographic data}

Doppler echocardiography was performed using commercially available equipment (Vivid 9 system, General Electric, Horten, Norway) and a variable frequency phased-array transducer. Complete M-mode, two-dimensional, spectraland color-Doppler images were used to obtain the following measurements: left atrial diameter (LAD), left ventricular end systolic diameter (LVESD), left ventricular end diastolic diameter (LVEDD), interventricular septal thickness (IVST), left ventricular posterior wall thickness (LVPWT), left ventricular ejection fraction (LVEF), pulmonary artery pressure (PAP). All measurements were taken according to the recommendations of the American Society of Echocardiography. Left ventricular ejection fractions were derived from biplane apical 2 and 4-chamber views using the modified Simpson's rule algorithm [23, 24].

TEE images were acquired with a $6 \mathrm{~T}$ phased array multiplane transoesophageal probe (Fig. 1a). LA and LAA were imaged in different tomographic planes from $0^{\circ}$ to $180^{\circ}$ to detect the presence of thrombusor SEC. LA thrombus was diagnosed by the presence of an echo-dense mass in left atrium or LAA (Fig. 1b), distinct from bulky pectinate muscles [25] (Fig. 1c). Left atrial spontaneous echocardiographic contrast (LASEC) as prethrombotic state having strong correlation with the occurrence of stroke was defined as a pattern of characteristic dynamic smoke-like swirling echoes in LA or LAA (Fig. 1d), distinct from a white noise artifact in the atrial cavity [26]. A pulsed Doppler sample was used to assess the Left atrial appendage flow velocities (LAAFV) [23]. Maximum emptying and filling velocities were estimated from an average of five well-defined emptying and filling waves. Left upper pulmonary venous flow velocity (LUPVFV) was assessed with a pulsed Doppler sample placed 1-2 $\mathrm{cm}$ into the left upper pulmonary vein proximal to where it enters the left atrium and at an angle as parallel as possible to the direction of the blood flow from the short-axis view obtained by advancing the TEE transducer to approximately $30 \mathrm{~cm}$ from the incisors [27, 28]. In addition, LAD was also obtained by TEE in the $45^{\circ}$ plane.

\section{Classification of left atrial appendage lobes}

The definition of a lobe includes following criteria: [i] a visible outpouching part demarcated by an external crease from the body of LAA; [ii] the inner diameter was at least 2-mm; [iii] the direction of lobe could be opposite with the main tubular body of LAA; [iv] the anatomic plane was occasionally but not necessarily lain in a same anatomic plane than the main tubular body; [v] the LAA had at least one lobe [29, 30]. Figure 2 showed the morphology of a LAA, the distinct protrusion parts represent lobes. All relevant measurements during TEE and TTE, LAT, LASEC and LAA lobes were confirmed by two experienced cardiologists, who were blinded to the study.

\section{Statistical analysis}

All statistical analyses were performed by SPSS for Windows version 22 (SPSS Inc., Chicago, IL). All continuous data are presented as the mean \pm SD and were compared using Student's t-test and one-way analysis of variance (ANOVA) test for two-level and multiple level grouping variables, respectively. Categorical variables are described as count and percent and were compared using Pearson's chi-square test (or Fisher's exact test whenever needed). Then sub-analysis based on gender and different numbers of LAA lobes was used to explore the 

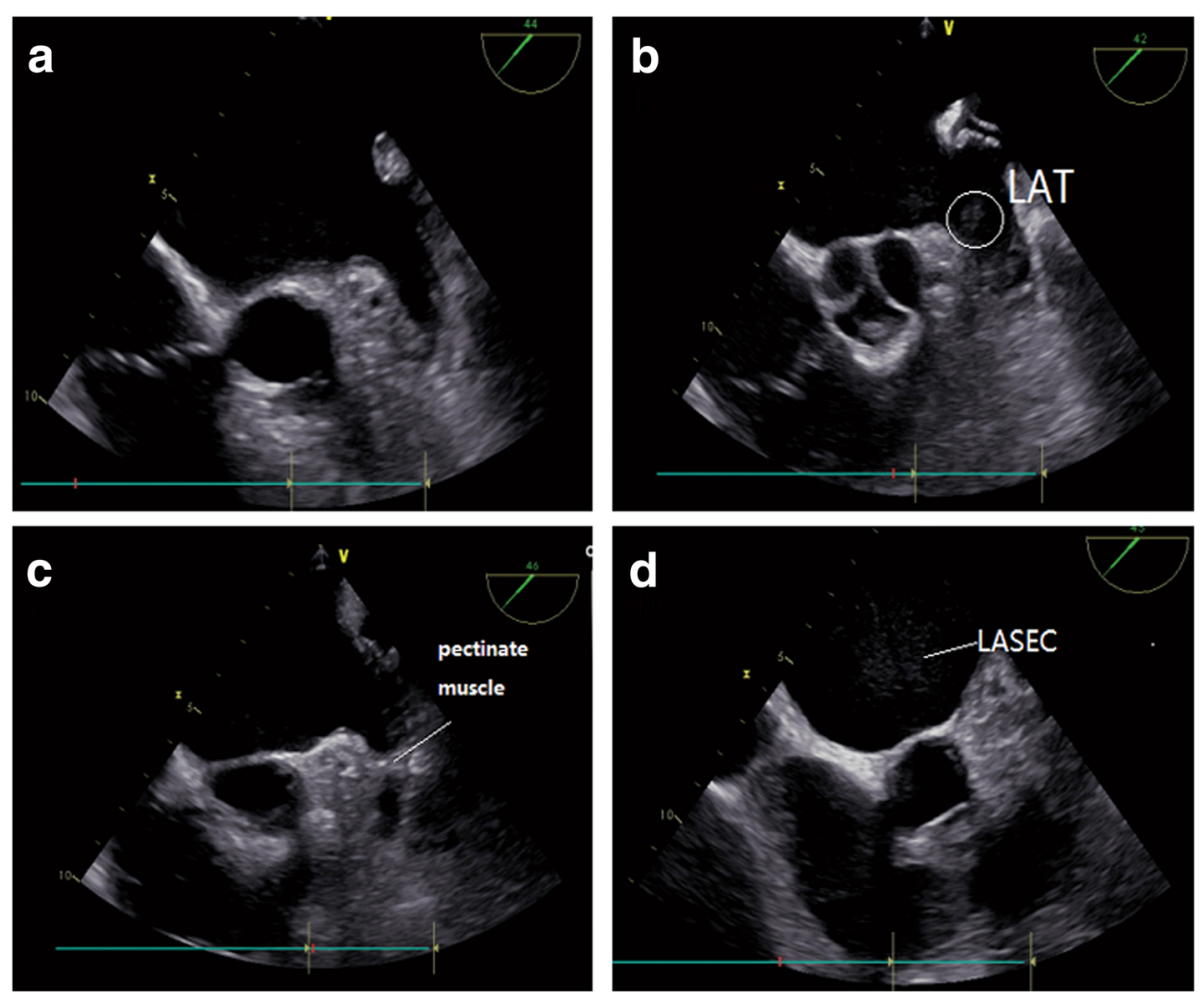

Fig. 1 Echo-pattern of LA or LAA thrombosis. The LAA regions are illustrated in a 2D TEE view (45-degree plane). a A normal image of left atrium and left atrial appendage (LAA) with one lobe. $\mathbf{b}$ A mass of thrombus about $9 \times 11 \mathrm{~mm}^{2}$ in the left atrium near the LAA orifice. $\mathbf{c}$ A thick pectinate muscle within the LAA cavity, distinct from LAA thrombus. $\mathbf{d}$ Left atrial spontaneous echo contrast (LASEC) presents as smoke-like swirling echoes
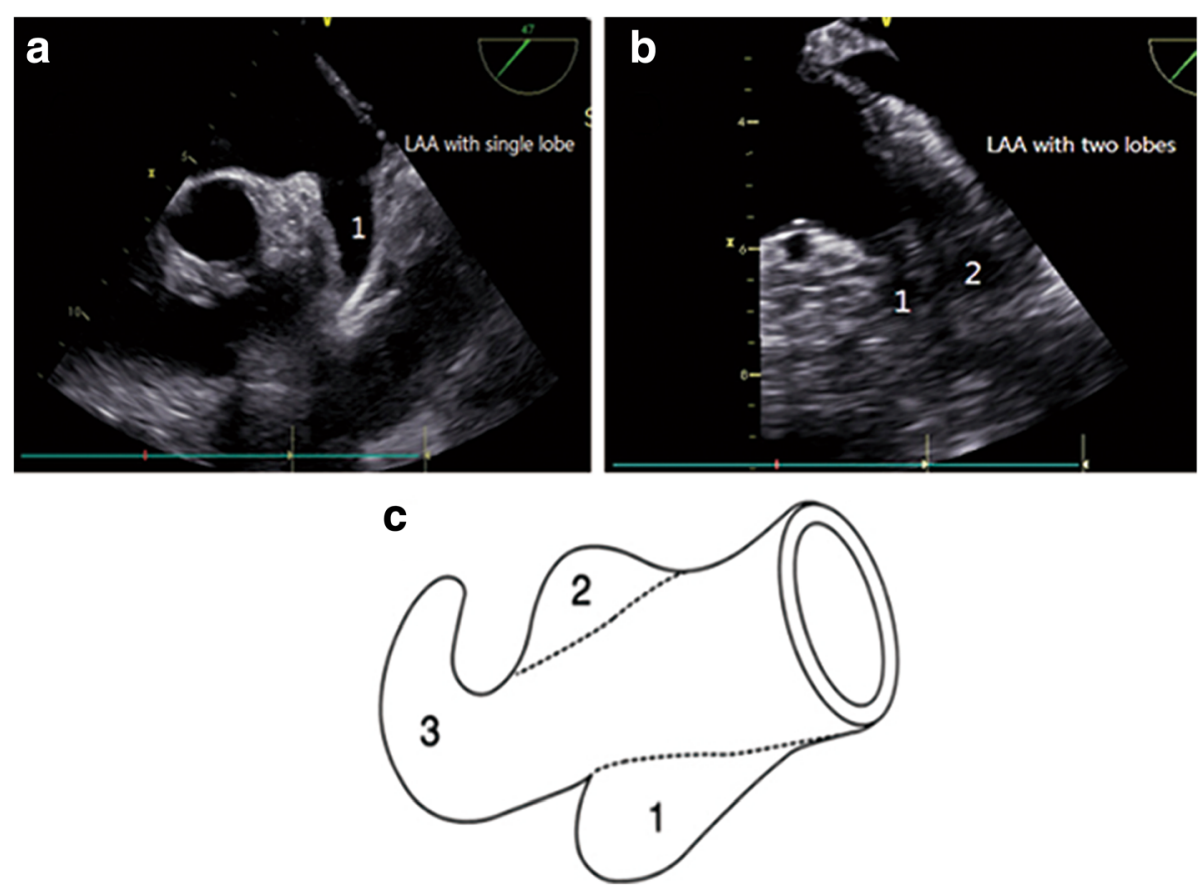

Fig. 2 Ultrasound images of LAA with different lobes by TEE. a LAA with one wide and deep lobe, composed by a tubular body and a blind-ending sac. b LAA with double lobes having shorts shape. $\mathbf{c}$ Diagram of a left atrial appendage (LAA) shows lobes (1, 2, and 3) 
correlation between LAA lobes and LAT/LASEC, the same as LUPVFV. The receiver operating characteristic (ROC) curve was used to discriminate the power of the LA anteroposterior diameter measured by TTE and TEE in identifying TEE surrogate markers of stroke (LA/LAA thrombus, LASEC) [23]. Comparisons of areas under ROC curves (AUC) were performed between the two measurements, using z-test. Finally, a multivariable logistic regression analysis model was used to identify the significant independent correlates of LAT or LASEC. All potentialco-founders were put into the model on the basis of known clinical relevance or statistically significant association observed in univariate analyses. The odds ratio (OR) and 95\% confidence interval (CI) of OR for thrombosis were computed. All tests were 2 -sided, and a $P$ value $<0.05$ was considered statistically significant.

\section{Results}

\section{Study population}

Baseline characteristics of 472 patients were shown in Table 1. LAT or LASEC were found in 80 patients (16.95\%, LAT: $n=23$; LASEC: $n=57$ ) by TEE examination. As shown in the table, patients with LAT or LASEC were significantly older but with nearly equal heart rate, systolic blood pressure (SBP), diastolic blood pressure (DBP) and without significant predilection for gender. The percentage distribution of prior congestive heart failure, diabetes, hypertension and previous vascular disease were significantly different between 2 patient groups, more in LAT/LASEC group. Simultaneously, patients with thrombosis had a longer duration of AF and more previous stroke events compared to patients without thrombosis; but these differences were statistically insignificant. In addition, persistent AF during hospitalization was more frequent and $\mathrm{CHA}_{2} \mathrm{DS}_{2}$-VASc score as expected, significantly higher in patients with LAT/LASEC.

\section{Laboratory examinations and echocardiographic characteristics}

These patients' baseline laboratory examinations and echocardiographic characteristics were shown in Table 2. There were no significant differences between 2 groups in platelets, ALT, AST, serum creatinine, BUN, D-dimer, and it was the same with LVESD, LVEDD, LVPWT, LAAFV and PFO by TTE. However, patients with LAT/ LASEC had lower hemoglobin level $(130.7 \pm 13.1$ vs $134.0 \pm 18.6, P=.049)$, larger LA anteroposterior diameter measured by TTE $(48.1 \pm 7.7$ vs $44.6 \pm 6.5, P<.001)$ or TEE (52.2 \pm 6.2 vs $46.7 \pm 7.1, \mathrm{P}<.001)$, slightly thicker ventricular septal $(10.7 \pm 3.0$ vs $9.8 \pm 1.7, P=.018)$, lower LVEF $(60.3 \pm 9.3$ vs $63.5 \pm 8.8, P=.005)$, higher pulmonary artery pressure $(30.9 \pm 9.8$ vs $28.1 \pm 8.2, P<.011)$,

Table 1 Baseline characteristics of included patients with and without LAT/LASEC

\begin{tabular}{|c|c|c|c|c|}
\hline & $\begin{array}{l}\text { Total } \\
(n=472)\end{array}$ & $\begin{array}{l}\text { LAT /LASEC } \\
\text { group }(n=80)\end{array}$ & Non-LAT/LASEC group $(n=392)$ & $P$ value \\
\hline \multicolumn{5}{|l|}{ Clinical characteristics } \\
\hline Age(yrs) & $66.1 \pm 10.8$ & $70.2 \pm 9.6$ & $65.3 \pm 10.8$ & $<0.001^{*}$ \\
\hline Male (n, \%) & $271(57.4 \%)$ & $40(50.0 \%)$ & $231(58.9 \%)$ & 0.154 \\
\hline$H R$ & $86.5 \pm 21.8$ & $86.8 \pm 18.9$ & $86.4 \pm 22.3$ & 0.895 \\
\hline SBP & $134.1 \pm 18.8$ & $137.0 \pm 21.7$ & $133.4 \pm 18.1$ & 0.136 \\
\hline DBP & $78.7 \pm 11.7$ & $79.3 \pm 13.1$ & $78.6 \pm 11.3$ & 0.642 \\
\hline Persistent AF (n, \%) & $154(32.6 \%)$ & $44(55.0 \%)$ & $107(27.3 \%)$ & $<0.001^{*}$ \\
\hline Duration of symptoms (months) & $45.0 \pm 70.4$ & $56.6 \pm 76.1$ & $42.4 \pm 69.0$ & 0.131 \\
\hline Congestive heart failure (n, \%) & $73(15.5 \%)$ & $24(30 \%)$ & 49 (12.5\%) & $0.003^{*}$ \\
\hline Hypertension (n, \%) & $277(58.7 \%)$ & $63(78.8 \%)$ & $214(54.6 \%)$ & $<0.001^{*}$ \\
\hline Diabetes mellitus (n, \%) & $77(16.3 \%)$ & $20(25.0 \%)$ & $57(14.5 \%)$ & 0.065 \\
\hline Previous stroke (n, \%) & $119(25.2 \%)$ & $26(32.5 \%)$ & $93(23.7 \%)$ & 0.174 \\
\hline Vascular disease (n, \%) & $133(28.2 \%)$ & $32(40.0 \%)$ & $101(25.8 \%)$ & $0.045^{*}$ \\
\hline CHA2DS2-VASc score & $2.86 \pm 1.81$ & $3.79 \pm 1.75$ & $2.65 \pm 1.76$ & $<0.001^{*}$ \\
\hline \multicolumn{5}{|l|}{ Antiplatelet and Anticoagulant drugs } \\
\hline Aspirin & $142(30.1 \%)$ & 15 (18.8\%) & $127(32.4 \%)$ & $0.033^{*}$ \\
\hline Warfarin & $187(39.6 \%)$ & $37(46.3 \%)$ & $150(38.3 \%)$ & \\
\hline Dabigatran & $47(10.0 \%)$ & $13(32.5 \%)$ & $34(8.7 \%)$ & \\
\hline Rivaroxaban & $2(0.42 \%)$ & 0 & $2(0.51 \%)$ & \\
\hline
\end{tabular}

Values are mean \pm standard deviation, or number (\%)

$L A T$ left atrial thrombus, $L A S E C$ left atrial appendage spontaneous echo contrast, $A F$ atrial fibrillation, $H R$ heart rate, $S B P$ systolic blood pressure, $D B P$ diastolic blood pressure. ${ }^{*} P<0.05$ 
Table 2 Laboratory examinations and echocardiographic characteristics of thrombosis group and control group

\begin{tabular}{|c|c|c|c|c|}
\hline & $\begin{array}{l}\text { Total } \\
(n=472)\end{array}$ & $\begin{array}{l}\text { LAT /LASEC } \\
\text { group }(n=80)\end{array}$ & Non-LAT/LASEC group $(n=392)$ & $P$ value \\
\hline \multicolumn{5}{|l|}{ Laboratory examinations } \\
\hline $\mathrm{Hb}(\mathrm{g} / \mathrm{l})$ & $133.8 \pm 17.8$ & $130.7 \pm 13.1$ & $134.0 \pm 18.6$ & $0.049^{*}$ \\
\hline Plt $(* 10 \wedge 9 / l)$ & $186.9 \pm 61.6$ & $183.0 \pm 54.3$ & $187.7 \pm 63.2$ & 0.576 \\
\hline $\mathrm{ALT}(\mathrm{U} / \mathrm{L})$ & $28.6 \pm 61.0$ & $22.6 \pm 21.1$ & $30.0 \pm 66.7$ & 0.361 \\
\hline $\mathrm{AST}(\mathrm{U} / \mathrm{L})$ & $29.7 \pm 75.7$ & $24.1 \pm 13.8$ & $30.9 \pm 83.9$ & 0.508 \\
\hline $\mathrm{sCr}(\mathrm{mmol} / \mathrm{l})$ & $81.3 \pm 25.0$ & $85.7 \pm 24.8$ & $80.2 \pm 25.0$ & 0.102 \\
\hline BUN (mmol/l) & $6.3 \pm 2.1$ & $5.7 \pm 1.7$ & $6.2 \pm 2.1$ & 0.167 \\
\hline D-Dimer & $0.53 \pm 1.67$ & $0.48 \pm 0.43$ & $0.54 \pm 1.62$ & 0.700 \\
\hline \multicolumn{5}{|c|}{ Transthoracic echocardiographic parameters } \\
\hline $\mathrm{LAD}(\mathrm{mm})$ & $45.2 \pm 6.8$ & $48.1 \pm 7.5$ & $44.6 \pm 6.5$ & $<0.001^{*}$ \\
\hline LVESD (mm) & $30.6 \pm 5.6$ & $31.6 \pm 5.3$ & $30.4 \pm 5.6$ & 0.092 \\
\hline $\operatorname{LVEDD}(\mathrm{mm})$ & $47.4 \pm 5.0$ & $47.5 \pm 5.1$ & $47.3 \pm 4.5$ & 0.755 \\
\hline IVsT (mm) & $9.96 \pm 1.98$ & $10.67 \pm 3.00$ & $9.79 \pm 1.67$ & $0.018^{*}$ \\
\hline LVPWT (mm) & $9.58 \pm 1.60$ & $10.26 \pm 2.44$ & $9.42 \pm 1.33$ & 0.418 \\
\hline LVEF (\%) & $63.0 \pm 9.0$ & $60.3 \pm 9.3$ & $63.5 \pm 8.8$ & $0.005^{*}$ \\
\hline Pulmonary artery pressure $(\mathrm{mmHg})$ & $28.6 \pm 8.5$ & $30.9 \pm 9.8$ & $28.1 \pm 8.2$ & $0.011^{*}$ \\
\hline \multicolumn{5}{|c|}{ Transesophageal echocardiographic parameters } \\
\hline $\mathrm{LAD}(\mathrm{mm})$ & $47.6 \pm 7.3$ & $52.2 \pm 6.2$ & $46.7 \pm 7.1$ & $<0.001^{*}$ \\
\hline Lobes of Left atrial appendage & $1.32 \pm 0.58$ & $1.67 \pm 0.77$ & $1.25 \pm 0.50$ & $<0.001^{*}$ \\
\hline $1(n, \%)$ & $289(61.2 \%)$ & $47(41.3 \%)$ & $242(78.3 \%)$ & $<0.001^{*}$ \\
\hline $2(n, \%)$ & $83(17.6 \%)$ & $25(31.3 \%)$ & $58(18.8 \%)$ & \\
\hline$>=3(n, \%)$ & $17(3.6 \%)$ & $8(10.0 \%)$ & 9 (2.9\%) & \\
\hline LUPVFV (m/s) & $0.63 \pm 0.24$ & $0.54 \pm 0.17$ & $0.67 \pm 0.26$ & $0.03^{*}$ \\
\hline LAAFV (m/s) & $0.35 \pm 0.19$ & $0.31 \pm 0.13$ & $0.37 \pm 0.20$ & 0.217 \\
\hline PFO (n, \%) & $33(7.0 \%)$ & 7 (8.8\%) & $26(6.6 \%)$ & 0.532 \\
\hline
\end{tabular}

Values are mean \pm standard deviation, or number (\%)

LAT left atrial thrombus, LASEC left atrial appendage spontaneous echo contrast, $A F$ atrial fibrillation, $H b$ hemoglobin, PLT platelets, $A L T$ glutamate pyruvate transaminase, $A S T$ glutamicoxaloacetic transaminase, $s C r$ serum creatinine, $B U N$ blood urea nitrogen, $L A D$ left atrial diameter, $L V E S D$ left ventricular end systolic diameter, LVEDD left ventricular end diastolic diameter, IVST Interventricular septal thickness, LVPWT left ventricular posterior wall thickness, LVEF left ventricular ejection fraction, LAAFV left atrial appendage flow velocity, PFO Patent foramen ovale. ${ }^{*} P<0.05$

more LAA lobes $(1.67 \pm 0.77$ vs $1.25 \pm 0.50, P<.001)$ and lower LUPVFV $(0.54 \pm 0.17 \mathrm{~m} / \mathrm{s}$ vs $0.67 \pm 0.26 \mathrm{~m} / \mathrm{s}, P$ <.011). Importantly, both T-test and Chi-Square test showed that LAA lobes of patients with LAT/LASEC were significantly more than other patients (Table 2).

\section{LA anteroposterior diameter measurements for identifying LAT/LASEC on TEE}

ROC analysis demonstrated that a cut-off value of anteroposterior LAD with 44.5 mmby TTEcould predict the presence of LAT/LASEC. At this level, sensitivity was $76.3 \%$ and specificity was $51.2 \%$, Area under the curve $(\mathrm{AUC})=0.67$ (CI 95\% 0.61-0.73, $p<0.001)$. The value of LAD by TEE with a sensitivity of $72.2 \%$ and a specificity of $63.6 \%$ was $48.5 \mathrm{~mm}$, AUC $=0.73$ (CI 95\% $0.67-0.79, p<0.001$ ) (Fig. 3 and Table 3 ). Thus, LAD by TTE and TEE showed a moderately high discriminatory power of the prediction of LAT/LASEC. However, ROC curve comparison for these two measurements revealed anteroposterior LAD by TEE provided greater predicative value than TTE with a significant difference (difference in AUC $=0.06 \pm 0.03, \mathrm{Z}=1.99, P<0.05)$.

\section{Correlation between the number of LAA lobes and prevalence of LAT/LASEC}

In our study, distribution of LAA lobes number was from 1 to 4 , consistent with previous studies [9]. The most frequent LAA $(61.2 \%)$ was a single lobe. The number of double and multiple lobes LAA types was 83 (17.6\%) and 17 (3.6\%). According to ANOVA, patients showed typical differences in LA thrombosis by TEE depending on LAA lobes number: among patients with LAT/LASEC by TEE, the average number of LAA lobes was $1.67 \pm 0.77$, compared with $1.25 \pm 0.50$ among patients with non-LAT/ 

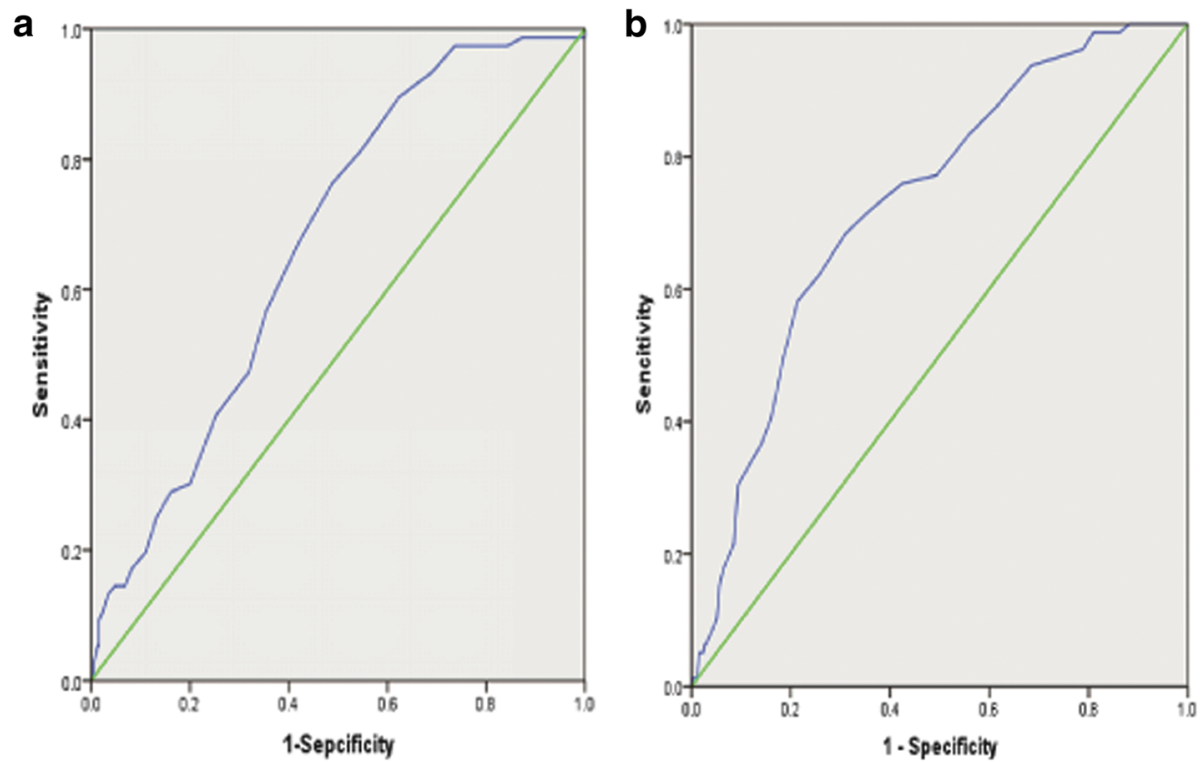

Fig. 3 Receiver operating characteristic curve (ROC) of $L A D$ for predicting the presence of $L A T / L A S E C$ in NVAF. a ROC analysis of $L A D$ measured by TTE for identifying LAT/LASEC; $\mathbf{b}$ ROC analysis of LAD measured by TEE for identifying LAT/LASEC

LASEC $(P<0.001)$ (Table 2). Moreover, LAT/LASEC incidence rate increased from single lobe to double and further to multiple lobes in sub-analysis in both male $(P<$ $0.001)$ and female $(P=0.004)$ subgroup (Fig. 4$)$.

\section{The correlation between LUPVFV and prevalence of LAT/ LASEC}

Overall, 95 patients (66 in LAT/LASEC group and 29 in non-LAT/LASEC group) had been detected LUPVFV by TEE. The average LUPVFV was $0.63 \pm 0.24 \mathrm{~m} / \mathrm{s}$. According to analysis of variance, LUPVFV decreased in patients with LAT/LASEC, compared with non-thrombosis patients (difference in means $0.13 \mathrm{~m} / \mathrm{s}, 95 \%$ CI 0.10 $0.22, P=0.032$ ) (Table 2). In sub-analysis, these patients were divided into four grades by quartiles. LAT/LASEC incidence rate decreased from first quartile to last quartile, but did not reached statistical significance in both male $(p=0.78)$ and female $(p=0.12)$ subgroup (Fig. 5).

\section{Independent predictive factors for LAT or LASEC}

Multiple candidate clinical predictors and echocardiography measurements were assessed as univariate independent predictors for LAT/LASEC. Our results

Table 3 The area under ROC curve of LAD to predict LAT/LASEC

\begin{tabular}{lllll}
\hline & AUC & SE & $P$ value & $95 \% \mathrm{Cl}$ \\
\hline LAD measured by TTE & 0.670 & 0.030 & $<.001$ & $0.611-0.730$ \\
LAD measured by TEE & 0.729 & 0.029 & $<.001$ & $0.672-0.787$
\end{tabular}

$R O C$ receiver operating characteristic, $L A D$ left atrial anteroposterior diameter, LAT left atrial thrombus, LASEC left atrial appendage spontaneous echo contrast, TTE transthoracic echocardiography, TEE transesophageal echocadiography, AUC area under ROC curve, SE standard error, $\mathrm{Cl}$ confident interval demonstrated that age, types of AF, LAD on TTE or TEE, antiplatelet or anticoagulation therapy, as well as $\mathrm{CHA}_{2} \mathrm{DS}_{2}$-VASc score were significantly correlated with the presence of LAT/LASEC (Tables 1 and 2). Multivariate linear regression analysis was performed to determine the relative importance of independent predictors of LAT/LASEC, the number of LAA lobes (single, double, multiple, odds ratio $2.37 ; 95 \% \mathrm{CI}$ $1.37-4.09 ; P=0.002$ ), AF types (paroxysmal, persistent, odds ratio $3.57 ; 95 \%$ CI $1.68-7.57 ; P=0.001)$ and antiplatelet or anticoagulation therapy (aspirin, oral coagulation medicine, odds ratio 0.36 ; $95 \%$ CI $0.13-$ $0.96 ; P=0.04$ ) were independent predictors of LAT/ LASEC (Table 4).

\section{Discussion}

In the present study, we demonstrated that more LAA lobes number were significantly and independently associated with the presence of LAT and LASEC. Another important finding was that patients with LA thrombosis had a lower LUPVFV. To the best of our knowledge, this is the first study to investigate the roles of LAA lobes number and LUPVFV in predicting left atrial stasis markers: LAT or LASEC.

Accumulative data documented that the presence of thrombus or SEC in LAA/LA are strongly associated with thromboembolism and adverse outcomes in NVAF patients [31-33]. Accordingly, TEE was recommended to evaluate the risk of thromboembolism previous to procedures such as cardioversion, catheter ablation or left atrial appendage closure (LAAC) [1, 34, 35]. In our study, the presence of LAT or LASEC was associated 


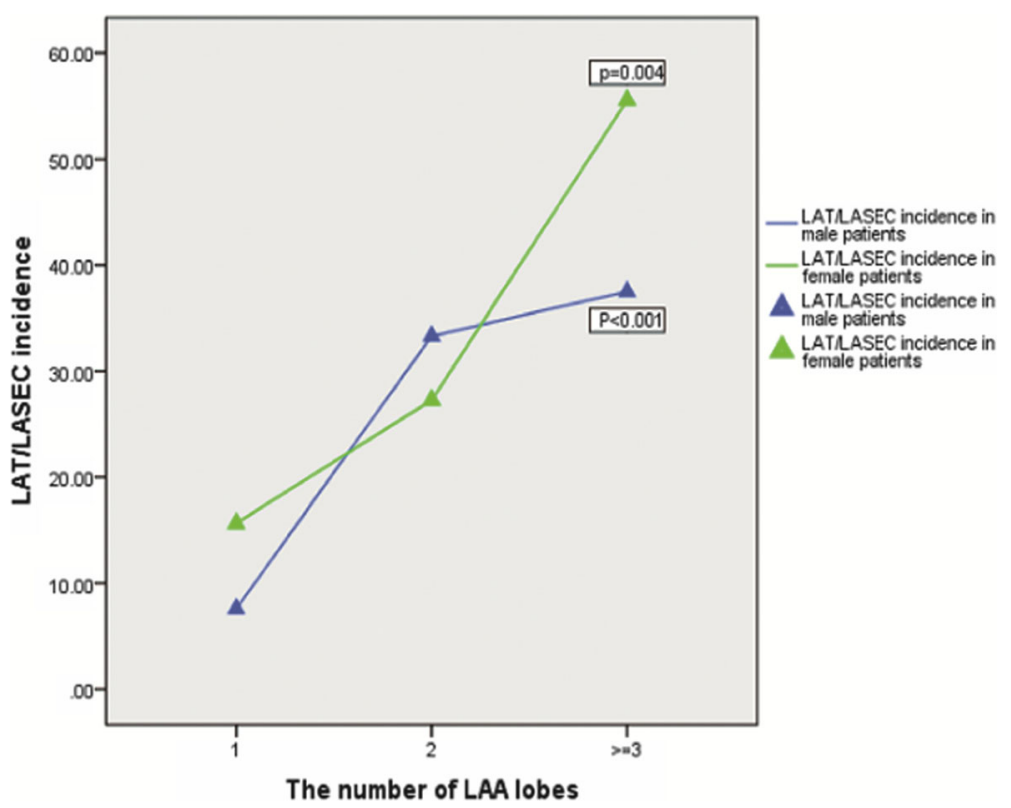

Fig. 4 Correlation between left atrial thrombosis and the number of LAA lobes. According to sub-analysis, LAT/LASEC differs significantly among different numbers of LAA lobes in either male $(P<0.001)$ or female $(P=0.004)$ subgroup. Patients with single lobe $L A A$ show a reduced prevalence of thrombus and SEC during TEE compared with patients with multilobe

with the risk of thromboembolism assessed by $\mathrm{CHA}_{2} \mathrm{DS}_{2}$-VASc score, due to a higher prevalence of recognized thromboembolic risk factors such as elder, congestive heart failure, vascular disease, hypertension, and diabetes. Furthermore, our results also indicated that LAT or LASEC was closely associated with a larger left atrium, independent of detection methods, such as TTE or TEE, as reported in previous studies [11, 3638]. However, this association became not significant after adjustment for other potential co-founders. What's

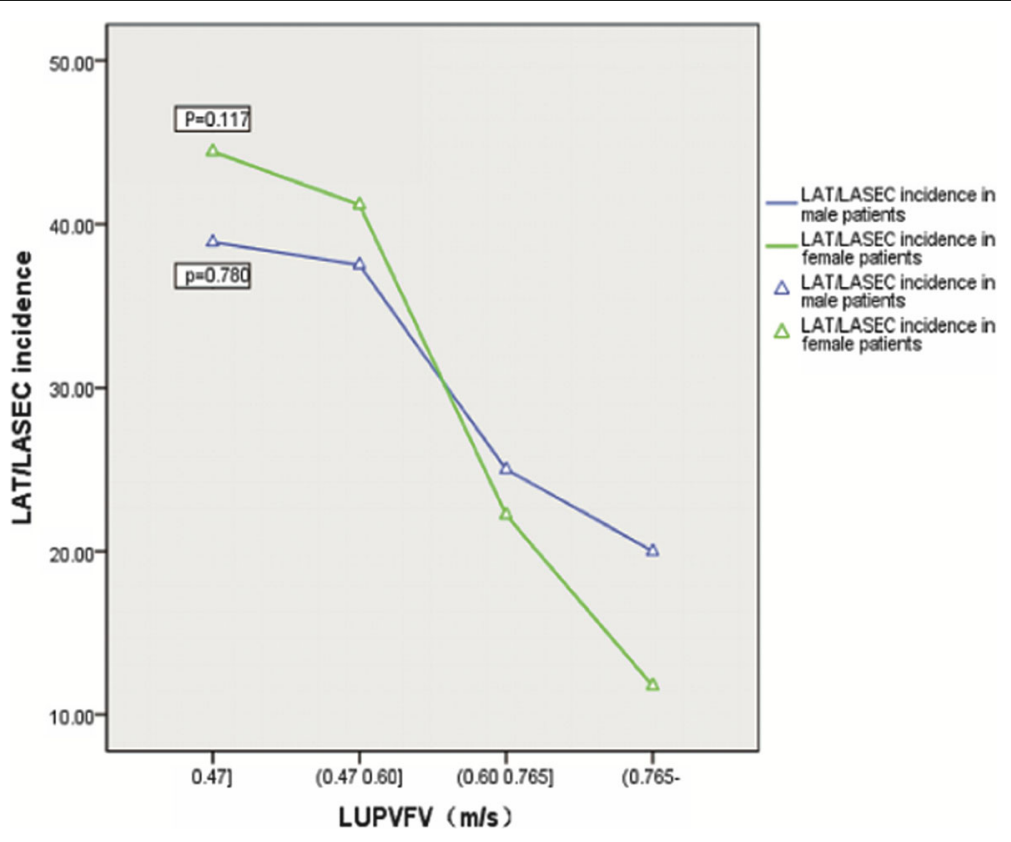

Fig. 5 Correlation between left atrial thrombosis and left upper pulmonary venous flow velocity (LUPVFV).The patients in our study who had been detected LUPVFV during TEE were divided into four groups by quartiles. In sub-analysis, LUPVFV decelerated, and the incidence of LAT/LASEC rose gradually from the last quartile to the first quartile, but did not reached statistical significance in both male $(p=0.78)$ and female $(p=0.12)$ subgroup owing to the small sample size 
Table 4 Multivariate logistic regression analysis on predictors of LAT or LASEC in patients with NVAF

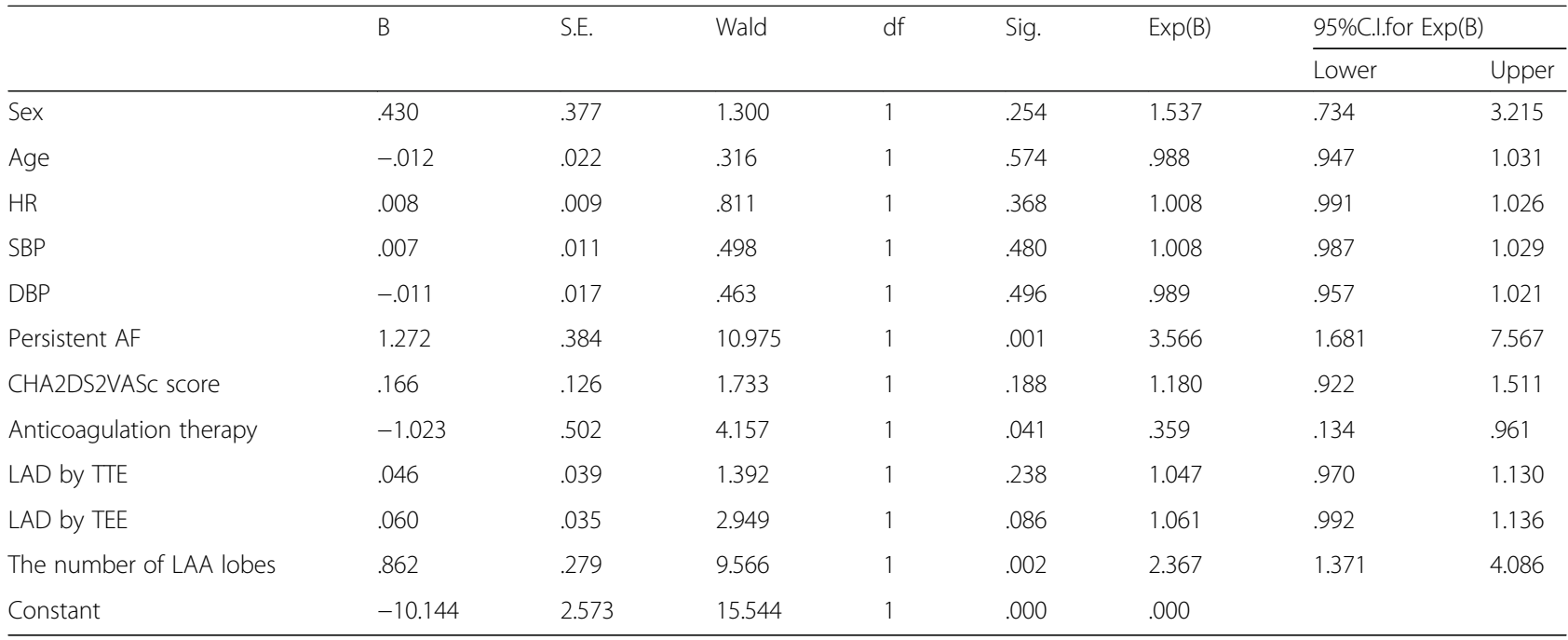

LAT left atrial thrombus, $L A S E C$ left atrial appendage spontaneous echo contrast, $A F$ atrial fibrillation, $H R$ heart rate, $S B P$ systolic blood pressure, $D B P$ diastolic blood pressure, $L A D$ left atrial diameter, $T E$ transthoracic echocardiography, TEE transesophageal echocadiography, $L A A$ left atrial appendage

more, our result showed that LAD by TEE provided greater predicted value than by TTE. Although, recent researchers found that LA area in four-chamber view, indexed area-length volumes and diastolic function parameters (E/e' and e' velocity) displayed strong correlation with left atrial stasis markers (LAT, LASEC, LAAFV $<20 \mathrm{~cm} / \mathrm{s}$ ) in patients with non-valvular AF [11, 39], our study limited by missing data couldn't be conducted in these aspects.

Previous studies indicated that the number of lobes was variable, with the prevalence of single lobe ranging from 20 to $42.7 \%$, double lobes from 25.2 to $64.3 \%$, multiple lobes from 26.0 to $35.7 \%$ [5, 18, 30]. However, our result showed that patients with a single lobe were in the majority(61.2\%), and double lobes $17.9 \%$. These variations may be attributable to the race discrepancy, sample sizes of population and subjective judgment of LAA morphology.

LAA morphology was often classified into four types: (i) Chicken Wing LAA, a main lobe $(>4 \mathrm{~cm})$ with a folded angle under $100^{\circ}$; (ii) Windsock LAA, a main lobe $(>4 \mathrm{~cm})$ with a folded angle over $100^{\circ}$; (iii) Cactus LAA, a main lobe $(<4 \mathrm{~cm})$ with more than two lobes over $1 \mathrm{~cm}$; and (iv) Cauliflower LAA, a main lobe $(<4 \mathrm{~cm})$ with no forked lobes. Such a division of LAA morphology which was originally designed to help practical planning for a transcatheter LAA closure device placement is now widely recognized [40].According to the classification, a Chicken Wing LAA often has only one lobe, sometimes two, while multilobed LAA were more common in NON-Chicken Wing patients. A large number of studies reported an association between LAA morphologies and the risk of TIA and stroke, Chicken Wing LAA had a highest LAA emptying flow velocity and a lowest risk of TIA and stroke [16, 18]. These studies documented that LAA emptying flow and LAAFV decreased in multilobed LAA [18], whereas LAAFV was associated with thrombus formation and stroke, regarded as a predictor of LA thromboembolism [20, 41]. Thus, our results support the findings of previous studies: patient with complicated morphology (like non-chicken LAA) had more commonly multilobed LAA, lower LAAFV and was more likely to develop thrombus and SEC than patient with a single lobe LAA. Further research should explore the correlation between LAA lobes and LAAFV.

Additionally, it is generally believed that Chicken Wing LAA is similar to Windsock LAA, and it is also difficult to differentiate Cactus LAA from Cauliflower LAA by morphology. Therefore, such category method is subjective and conflicting. However, the number of LAA lobes by TEE is objective and easy to test, while cardiac CT and MRI are expensive and deleterious, thus we can apply TEE to acquire the number of LAA lobes and examine LAT and LASEC simultaneously.

Additionally, our study documented that left upper pulmonary venous flow velocity (LUPVFV) decreased in LAT/LASEC group. LUPVFV can reflect LA pressure and LV diastolic filling pressure $\left(E / e^{\prime}\right)$, which are important influential factors for LAAFV [27, 42]. Consequently, the combined use of LUPVFV and LAA lobes number can provide additional clinical implications for risk stratification.

\section{Study limitations}

This study has several limitations. First, this study is a single-center study with a relatively small sample. Second, the retrospective design of the study is an 
additional limitation. Large-scale studies with long-term follow-up are warranted to evaluate the predictive value of LAA lobes for stroke. Third, we investigated a relatively low risk population reflected by a mean $\mathrm{CHA}_{2} \mathrm{DS}_{2}$-VASc score of 2.86. Additionally, the rate of anticoagulation therapy among these patients was high (80.1\%), which may affect the rate of LAT and LASEC and thromboembolic events. Thus, our findings could not be adapted to a high-risk AF population.

\section{Conclusion}

More left atrial appendage lobes are associated with significantly higher risk of left atrial thrombus or left atrial spontaneous echo contrast in patients with non-valvular atrial fibrillation. Therefore, the number of LAA lobes is an independent risk factor and has a moderate predictive value for LAT/LASEC among NVAF patients in China.

\section{Abbreviations}

AF: Atrial fibrillation; CT: Computer tomography; LA: Left atrium; LAA: Left atrial appendage; LAAC: Left atrial appendage closure; LAAFV: Left atrial appendage flow velocity; LAD: Left atrial diameter; LASEC: Left atrial spontaneous echo contrast; LAT: Left atrial thrombus; LUPVFV: Left upper pulmonary venous flow velocity; LV: Left ventricle; LVEF: Left ventricular ejection fraction; MRI: Magnetic resonance imaging; NVAF: Non-valvular atrial fibrillation; TEE: Transesophageal echocardiography; TIA: Transient ischemic attack; TTE: Transthoracic echocardiography

\section{Funding}

This work was supported by the National Natural Science Foundation of China (81270194). The funders had no role in study design, data collection and analysis, decision to publish, or preparation of the manuscript.

\section{Availability of data and materials}

The dataset of this article is accessible on reasonable request from the corresponding author.

\section{Authors' contributions}

DX and YS2 conceived the study, FW, MZ, YS1, WZ, XP and DG acquired the data, FW and MZ performed and analyzed all echocardiograms, DX, FW and YS2 performed statistical analyses, DX, FW, YS2 and MZ drafted the manuscript, YW, WZ, YS2 and WC helped to draft the manuscript, and revised the manuscript critically for important intellectual content, XW, YL, YS1, XP, $D G, X Z$ and $Y X$ revised the manuscript critically for important intellectual content. All authors read, revised and accepted the final version of the manuscript. All authors read and approved the final manuscript.

\section{Ethics approval and consent to participate}

The study protocol was approved by Research Ethical Committee of Shanghai Tenth People's Hospital of Tongji University School of Medicine and conducted according to the principles expressed in the Declaration of Helsinki. The data was retrieved from hospital medical record system and therefore no additional informed consent was required. The institutional review board also waived the need for written informed consent from the participants. We guarantee that the patients' personal data have been secured.

\section{Consent for publication}

Written informed consents were obtained from these patients to use of their information and all the related images for scientific purpose.

\section{Competing interests}

The authors have declared that they have no competing interests.

\section{Publisher's Note}

Springer Nature remains neutral with regard to jurisdictional claims in published maps and institutional affiliations.

Received: 4 January 2018 Accepted: 16 July 2018

Published online: 31 July 2018

\section{References}

1. Fuster V, Rydén LE, Cannom DS, Crijns HJ, Curtis AB, Ellenbogen KA Halperin JL, Kay GN, Le Huezey JY, Lowe JE, et al. 2011 ACCF/AHA/HRS focused updates incorporated into the ACC/AHA/ESC 2006 Guidelines for the management of patients with atrial fibrillation: a report of the American College of Cardiology Foundation/American Heart Association Task Force on Practice Guidelines developed in partnership with the European Society of Cardiology and in collaboration with the European Heart Rhythm Association and the Heart Rhythm Society. J Am Coll Cardiol. 2011;57(11): e101-98.

2. Wilke T, Groth A, Mueller S, Pfannkuche M, Verheyen F, Linder R, Maywald $U$, Bauersachs R, Breithardt G. Incidence and prevalence of atrial fibrillation: an analysis based on 8.3 million patients. Europace. 2013;15(4):486-93.

3. Knecht S, Wilton SB, Haissaguerre M. The 2010 update of the ESC guidelines for the management of atrial fibrillation. Circ J. 2010;74(12):2534-7.

4. Furie KL, Goldstein LB, Albers GW, Khatri P, Neyens R, Turakhia MP, Turan TN Wood KA. Oral antithrombotic agents for the prevention of stroke in Nonvalvular atrial fibrillation: a science advisory for healthcare professionals from the American Heart Association/American Stroke Association. Stroke. 2012;43(12):3442-53.

5. Beigel R, Wunderlich NC, Ho SY, Arsanjani R, Siegel RJ. The left atrial appendage: anatomy, function, and noninvasive evaluation. JACC Cardiovasc Imaging. 2014;7(12):1251-65.

6. Sharma S, Devine W, Anderson RH, Zuberbuhler JR. The determination of atrial arrangement by examination of appendage morphology in 1842 heart specimens. Br Heart J. 1988;60(3):227-31.

7. Tuccillo B, Stümper O, Hess J, van Suijlen RJ, Bos E, Roelandt JR, Sutherland GR. Transoesophageal echocardiographic evaluation of atrial morphology in children with congental heart disease. Eur Heart J. 1992;13(2):223-31.

8. Zabalgoitia M, Leonard A, Blackshear JL, Safford R, Baker VS. Transesophageal echocardiographic correlates of thromboembolism in high-risk patients with nonvalvular atial fibrillation.The Stroke Prevention in Atrial Fibrillation Investigators Committee on Echocardiographyr. Ann Intern Med. 1998;128(8):639-47.

9. Fatkin D, Kelly RP, Feneley MP. Relations between left atrial appendage blood flow velocity, spontaneous echocardiographic contrast and thromboembolic risk in vivo. J Am Coll Cardiol. 1994;23(4):961-9.

10. Zabalgoitia M, Halperin JL, Pearce LA, Blackshear JL, Asinger RW, Hart RG. Transesophageal echocardiographic correlates of clinical risk of thromboembolism in nonvalvular atrial fibrillation. Stroke prevention in atrial fibrillation III investigators. J Am Coll Cardiol. 1998;31(7):1622-6.

11. Watson T, Shantsila E, Lip GY. Mechanisms of thrombogenesis in atrial fibrillation: Virchow's triad revisited. Lancet. 2009;373(9658):155-66.

12. Thambidorai SK, Murray RD, Parakh K, Shah TK, Black IW, Jasper SE, Li J, Apperson-Hansen C, Asher CR, Grimm RA, et al. Utility of transesophageal echocardiography in identification of thrombogenic milieu in patients with atrial fibrillation (an ACUTE ancillary study). Am J Cardiol. 2005;96(7):935-41.

13. Klein AL, Murray RD, Black IW, Chandra S, Grimm RA, DSa DA, Leung DY, Miller D, Morehead AJ, Vaughn SE, et al. Integrated backscatter for quantification of left atrial spontaneous echo contrast. J Am Coll Cardiol. 1996;28(1):222-31.

14. Fatkin D, Feneley MP. Qualitative or quantitative assessment of spontaneous echo contrast? J Am Coll Cardiol. 1997;29(1):222-4.

15. Bernhardt $P$, Schmidt $H$, Hammerstingl C, Omran H. Patients with atrial fibrillation and dense spontaneous echo contrast at high risk a prospective and serial follow-up over 12 months with transesophageal echocardiography and cerebral magnetic resonance imaging. J Am Coll Cardiol. 2005:45(11):1807-12.

16. Di Biase L, Santangeli P, Anselmino M, Mohanty P, Salvetti I, Gili S, Horton R, Sanchez JE, Bai R, Mohanty S, et al. Does the left atrial appendage morphology correlate with the risk of stroke in patients with atrial fibrillation? Results from a multicenter study. J Am Coll Cardiol. 2012;60:531-8. 
17. Vargas-Barrón J, Espinola-Zavaleta N, Roldán FJ, Romero-Cárdenas A, Keirns C, Vázquez-Antona C. Transesophageal echocardiographic diagnosis of thrombus in accessory lobes of the left atrial appendage. Echocardiography. 2000;17(7):689-91.

18. Petersen M, Roehrich A, Balzer J, Shin DI, Meyer C, Kelm M, Kehmeier ES. Leftatrial appendage morphology is closely associated with specific echocardiographic flow pattern in patients with atrial fibrillation. Europace. 2015;17(4):539-45.

19. Khurram IM, Dewire J, Mager M, Maqbool F, Zimmerman SL, Zipunnikov V, Beinart R, Marine JE, Spragg DD, Berger RD, et al. Relationship between left atrial appendage morphology and stroke in patients with atrial fibrillation. Heart Rhythm. 2013;10(12):1843-9.

20. Fukushima K, Fukushima N, Kato K, Ejima K, Sato H, Fukushima K, Saito C, Hayashi K, Arai K, Manaka T, et al. Correlation between left atrial appendage morphology and flow velocity in patients with paroxysmal atrial fibrillation. Eur Heart J Cardiovasc Imaging. 2016;17(1):59-66.

21. Sakr SA, El-Rasheedy WA, Ramadan MM, El-Menshawy I, Mahfouz E, Bayoumi M. Association between left atrial appendage morphology evaluated by trans-esophageal echocardiography and ischemic cerebral stroke in patients with atrial fibrillation. Int Heart J. 2015;56(3):329-34.

22. Providência R, Faustino A, Paiva L, Fernandes A, Barra S, Pimenta J, Trigo J, Botelho A, Leitão-Marques AM. Mean platelet volume is associated with the presence of left atrial stasis in patients with non-valvular atrial fibrillation. BMC Cardiovasc Disord. 2013;13:40.

23. Faustino A, Providência R, Barra S, Paiva L, Trigo J, Botelho A, Costa M, Gonçalves $L$. Which method of left atrium size quantification is the most accurate to recognize thromboembolic risk in patients with non-valvular atrial fibrillation? Cardiovasc Ultrasound. 2014;12:28.

24. Schiller NB, Acquatella H, Ports TA, Drew D, Goerke J, Ringertz H, Silverman $\mathrm{NH}$, Brundage $\mathrm{B}$, Botvinick $\mathrm{EH}$, Boswell $\mathrm{R}$, et al. Left ventricular volume from paired biplane two-dimensional echocardiography. Circulation. 1979;60(3): 547-55.

25. Beppu S, Park YD, Sakakibara H, Nagata S, Nimura Y. Clinical features of intracardiac thrombosis based on echocardiographic observation. Jpn Circ J. 1984;48(1):75-82.

26. Beppu S, Nimura Y, Sakakibara H, Nagata S, Park YD, Izumi S. Smoke-like echo in the left atrial cavity in mitral valve disease: its features and significance. J Am Coll Cardiol. 1985;6(4):744-9.

27. Li Y, Yuan LJ, Cao TS, Duan YY, Jia HP, Liu J. Effects of respiration on pulmonary venous flow and its clinical applications by Doppler echocardiography. Echocardiography. 2009;26(2):150-4.

28. Abdalla IA, Murray RD, Lee JC, White RD, Thomas JD, Klein AL. Does rapid volume loading during transesophageal echocardiography differentiate constrictive pericarditis from restrictive cardiomyopathy? Echocardiography. 2002;19(2):125-34.

29. Üçerler H, Ikiz ZA, Özgür T. Human left atrial appendage anatomy and overview of its clinical significance. Anadolu Kardiyol Derg. 2013;13(6): 566-72.

30. Veinot JP, Harrity PJ, Gentile F, Khandheria BK, Bailey KR, Eickholt JT, Seward JB, Tajik AJ, Edwards WD. Anatomy of the normal left atrial appendage: a quantitative study of age-related changes in 500 autopsy hearts: implications for echocariographic examination. Circulation. 1997; 96(9):3112-5.

31. Singer DE, Albers GW, Dalen JE, Go AS, Halperin JL, Manning WJ. Antithrombotic therapy in atrial fibrillation. The sixth ACCP conference on antithrombotic and thrombolytic therapy. Chest. 2004;126(3):429S-56S

32. Lip GY, Lane D, Van Walraven C, Hart RG. Additive role of plasma von Willebrand factor levels to clinical factors for risk stratification in patients with atrial fibrillation. Stroke. 2006;37(9):2294-300.

33. Zabalgoitia M, Halperin $J$, Pearce LA, Blackshear JL, Asinger RW, Hart RG. Transesophageal echocardiographic correlates of thromboembolism in high-risk patients with nonvalvular atrial fibrillation. J Am Coll Cardiol. 1998; 31(7):1622-6.

34. Camm AJ, Kirchhof P, Lip GY, Schotten U, Savelieva I, Ernst S, Van Gelder IC, Al-Attar N, Hindricks G, Prendergast B, et al. Guidelines for the management of atrial fibrillation: the task force for the management of atrial fibrillation of the European Society of Cardiology (ESC). Eur Heart J. 2010;31(19):2369-429.

35. Wunderlich NC, Beigel R, Swaans MJ, Ho SY, Siegel RJ. Percutaneous interventions for left atrial appendage exclusion options, assessment, and imaging using 2D and 3D echocardiography. JACC Cardiovasc Imaging. 2015;8(4):472-88.
36. Osranek M, Bursi F, Bailey KR, Grossardt BR, Brown RD, Kopecky SL, Tsang TS, Seward JB. Left atrial volume predicts cardiovascular events in patients originally diagnosed with lone atrial fibrillation: three-decade follow-up. Eur Heart J. 2005;26(23):2556-61.

37. Leung DY, Boyd A, Arnold H, Chi C, Thomas L. Echocardiographic evaluation of left atrial size and function: current understanding, pathophysiologic correlates, and prognostic implications. Am Heart J. 2008;156(6):1056-64.

38. Tang R, Dong J, Shang M, Du X, Yan X, Long D, Yu R, Wu J, Bai R, Liu N, et al. Impact of left atrium size on left atrial thrombus in patients with nonvalvular persistent atrial fibrillation. Zhonghua Yi Xue Za Zhi. 2015;95(14): 1083-7.

39. Doukky R, Garcia-Sayan E, Patel M, Pant R, Wassouf M, Shah S, D'Silva O, Kehoe RF. Impact of diastolic function parameters on the risk for left atrial appendage Thrombus in patients with Nonvalvular atrial fibrillation: a prospective study. J Am Soc Echocardiogr. 2016;29(6):545-53.

40. Wang Y, Di Biase L, Horton RP, Nguyen T, Morhanty P, Natale A. Left atrial appendage studied by computed tomography to help planning for appendage closure device placement. J Cardiovasc Electrophysiol. 2010; 21(9):973-82.

41. Handke M, Harloff A, Hetzel A, Olschewski M, Bode C, Geibel A. Left atrial appendage flow velocity as a quantitative surrogate parameter for thromboembolic risk: determinants and relationship to spontaneous echocontrast and thrombus formation--a transesophageal echocardiographic study in 500 patients with cerebral ischemia. J Am Soc Echocardiogr. 2005;18(12):1366-72.

42. Alnabhan N, Kerut EK, Geraci SA, McMullan MR, Fox E. An approach to analysis of left ventricular diastolic function and loading conditions in the echocardiography laboratory. Echocardiography. 2008;25(1):105-16.

\section{Ready to submit your research? Choose BMC and benefit from:}

- fast, convenient online submission

- thorough peer review by experienced researchers in your field

- rapid publication on acceptance

- support for research data, including large and complex data types

- gold Open Access which fosters wider collaboration and increased citations

- maximum visibility for your research: over $100 \mathrm{M}$ website views per year

At BMC, research is always in progress.

Learn more biomedcentral.com/submissions 